\title{
REUTILIZACIÓN DE UN EDIFICIO: HOTEL CONVENTO NUESTRA SEÑORA DE CHINQUINQUIRÁ
}

María Claudia Quiroz Uribe*

Imagen I. Vista del claustro e interior del Convento Nuestra Señora de Chiquinquirá Fuente: Fotografía de la autora

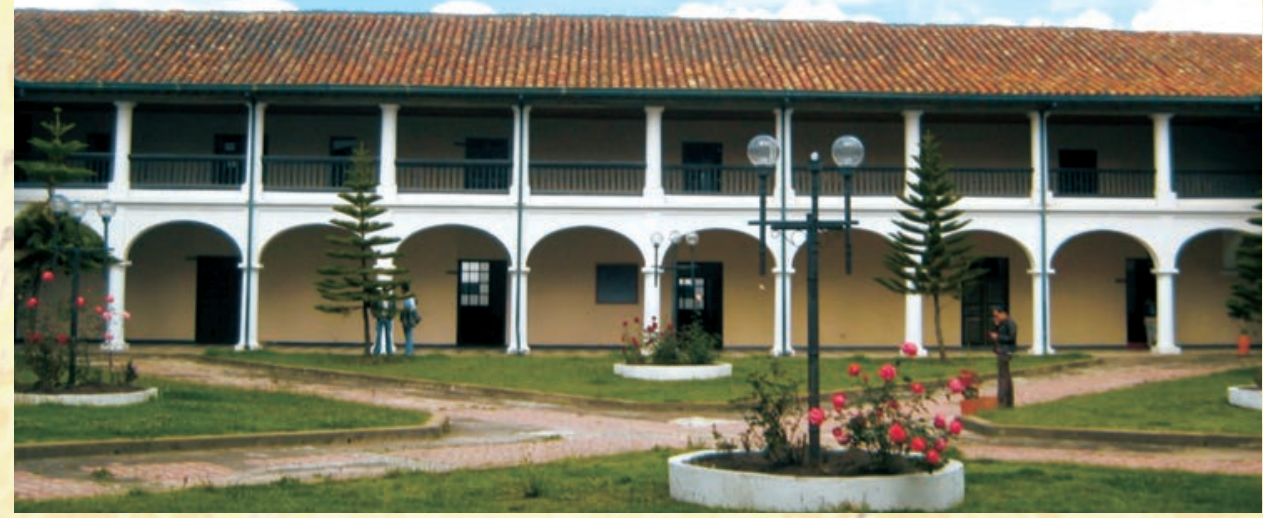

\section{RESUMEN}

Construido a principios del siglo XIX, el Convento Nuestra Señora de Chiquinquirá, ubicado en la ciudad de Chiquinquirá, departamento de Boyacá, conserva en gran parte sus condiciones físicas naturales, claustro de dos plantas en "u" y pabellones rodeados de corredores con arcadas. En este sentido, el proyecto plantea conservar las condiciones físicas actuales de la edificación, la cual por su valor histórico y urbano merece ser respetada, se plantea un nuevo uso el de "Hotel" en las dos plantas y su integración con un edificio moderno ubicado en un predio anexo, que permita cumplir con el programa del hotel de manera exitosa. De igual forma se pretende comprobar que los edificios tienen la capacidad de adaptarse a otros usos, para contribuir a la mitigación del desgaste energético ocasionado por las demoliciones de edificaciones que aún pueden ser utilizadas.

\section{PALABRAS CLAVE}

Convento Nuestra Señora de Chiquinquirá, Boyacá, Reutilización, Patrimonio, Hotel. 


\section{BUILDING REUSE: HOTEL CONVENT OUR LADY OF CHINQUINQUIRÁ}

María Claudia Quiroz Uribe*

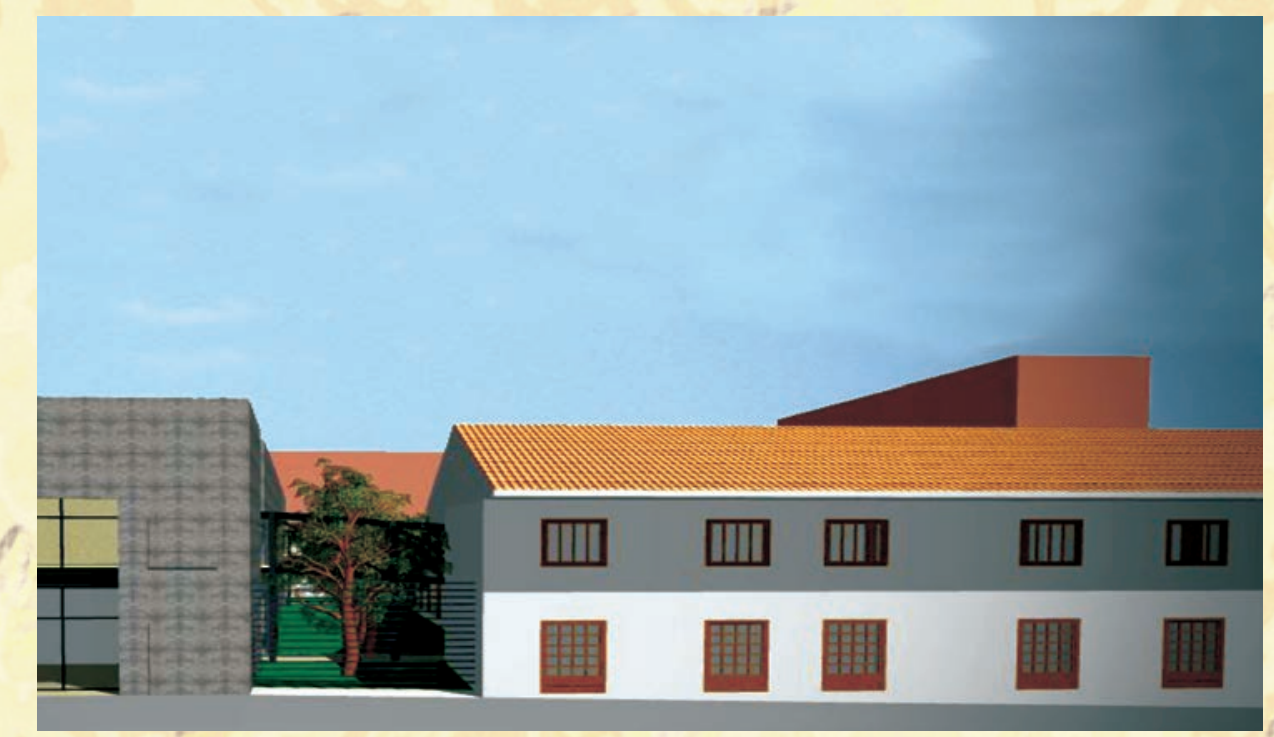

\section{ABSTRACT}

Built in the early nineteenth century, the Convent Our Lady of Chiquinquirá, located in the city of Chiquinquirá, Boyacá, largely preserve its natural physical conditions, two-story cloister "u" and pavilions surrounded by corridors with arcades. Therefore, the project proposes to conserve the current condition of the building, which for its historical and urban value deserves respect, so is projected a new use of "Hotel" at the two plants and its integration with a modern building on a property annex, which will allow accomplish the hotel program successfully. Besides, it is intended to prove that buildings have the ability to adapt to other uses, and contribute to the mitigation of loss of energy caused by the demolition of buildings that can still be used.

\section{KEY WORDS}

Convent Our Lady of Chiquinquirá, Boyacá, Reuse, Hotel, Heritage
* Este artículo es producto de la investigación realizada para optar por el título de arquitecta en la Facultad de Arquitectura de la Universidad Santo Tomás, seccional Bucaramanga, la cual fue calificada como meritoria.

** El proyecto fue dirigido por el arquitecto Hernando Ladino Barriga
Imagen 2. Render vista exterior Hotel Convento Nuestra Señora de Chiquinquirá. Fuente: Elaborado por la autora 


\section{INTRODUCCIÓN}

Es notable el deterioro que se ha dado del medio ambiente, debido a una gran cantidad de circunstancias que en su mayoría se gestan en los entornos urbanos. Para nadie es un secreto que las ciudades son las grandes productoras de $\mathrm{CO} 2$, y que el sector de la construcción contribuye en casi el $50 \%$ en su producción.

En respuesta a la situación anterior y con una mirada responsable respecto al desarrollo profesional de la arquitectura, se propone en el ejercicio académico la reutilización de una estructura existente, con la convicción de que esta determinación contribuye con la disminución de los impactos negativos generados en los procesos constructivos. Aunque no se trata de un tema abordado de forma masiva en la arquitectura colombiana, razón por la cual no se cuenta con suficiente información en nuestro medio al respecto, la práctica de intervenir un edificio existente, ha sido desarrollada de manera constante a lo largo de la historia de la humanidad. En consecuencia dicha intervención resulta como una respuesta a un problema funcional, de obsolescencia y seguramente la más importante: el edificio tiene implícito un valor histórico de apropiación por parte de su comunidad que lo vuelve patrimonio y, por lo tanto, resulta ser la razón más importante para conservarlo.

Tomar la decisión de reutilizar un edificio y la selección de éste en particular no es nada fácil, hay que tener en cuenta muchos aspectos que permiten que el proyecto sea viable, tanto desde el punto de vista económico como social, debido a que se interviene en un contexto que tiene una memoria colectiva de lo que ha sido el objeto arquitectónico. En este caso el proyecto se desarrolla sobre un convento que fue construido a principios del siglo XIX, ubicado en el municipio de Chiquinquirá, en el departamento de Boyacá, que cuenta con determinantes que lo califican para una reutilización.

\section{DEFINICIÓN DEL PROBLEMA}

Un edificio puede expresar en sí tantas cosas como el contexto en el que se encuentra inmerso, de allí que edificio y contexto se encuentren en constante relación y, por tanto, las intervenciones realizadas en un ámbito u otro los afecta mutuamente. Cuando un edificio se encuentra abandonado o en desuso, el sector en el cual se encuentra emplazado proyecta una imagen no siempre positiva y esta proyección es recibida como una realidad transversal que lo envuelve incluso hasta alcanzar un proceso de deterioro.

Esta situación además de presentarse como un problema que debe ser resuelto por la ciudad, debe contemplar el hecho de que el edificio por sí solo tiene un valor propio que lo ha adquirido gracias a sus características históricas (por la época de su construcción y la cantidad de situaciones que se gestaron allí), estéticas (por el reflejo de un estilo arquitectónico) y sociales (por el referente que se ha vuelto y es en la ciudad, atribución que le han dado sus ciudadanos), de allí que el edificio sea tan importante como el contexto en el cual se encuentra ubicado.

Además del contexto, es preciso atender otra problemática que radica en la forma como se debería intervenir para seguir los postulados de preservación del medio ambiente. Hay que tener claro que en la construcción de cualquier edificación se gasta gran cantidad de energía y, por tanto, es necesario tratar de alargar la vida útil de los edificios lo máximo posible, habida cuenta que éste debe presentar las capacidades necesarias para realizarlo. 
Cada día es más común ver que edificios que fueron proyectados para un uso específico se encuentran abandonados, consecuencia directa del constante cambio que presentan las sociedades y que se ve reflejado en el entorno construido. Es así que en el problema confluyen una serie de circunstancias físicas, constituidas por la afectación que hace el objeto arquitectónico obsoleto a la ciudad y a su sector inmediato; al igual que otras de tipo social en donde se ve el deterioro de un elemento de carácter histórico que hace parte de la memoria colectiva, con cuya revitalización se lograría afianzar el patrimonio cultural inmueble; así como también se precisa atender las de tipo ambiental por su consumo energético.

\section{JUSTIFICACIÓN}

La reutilización de un edificio existente se justifica desde varios puntos de vista: el re-uso de un edificio soluciona una gran cantidad de problemas de tipo ambiental en donde se perpetúa la continuidad y se excluye la posibilidad de destrucción que le hace mal al medio ambiente, en términos de gasto de energía, de producción de $\mathrm{CO} 2$ y de explotación de recursos no renovables. También se evita la erosión de la tierra, la creación de polución, no se pierde tanto tiempo y dinero en movilidad de materiales, y por último se reduce el costo de infraestructuras.

Desde una mirada morfológica del tejido urbano existente, la estrategia debería ser adaptar, transformar, y convertir, no como una oportunidad sino como una necesidad para conservar las características propias de cada espacio, en este caso de cada ciudad. Concentrándose en la situación particular del edificio que se pretende intervenir, el convento de Chiquinquirá, hace parte del centro histórico de la ciudad y, es de gran importancia por su legado en la construcción de país, también desde un punto de vista arquitectónico por su paisaje colonial predominante y desde el aspecto religioso del que hace parte. Estas razones, en consecuencia, generan una actividad turística que gira en torno de estos atractivos.

El edificio, por su ubicación e importancia, genera la necesidad de ser conservado, y de alguna forma puesto en uso a favor de la comunidad. Esta intervención en este objeto arquitectónico generará reacciones positivas, tanto para su contexto más próximo como para la ciudad en general, pues se espera con el proyecto lograr la revitalización del paisaje, al evidenciar la posibilidad que un edificio que fue construido hace casi 200 años, promueve y posibilita una actuación contemporánea, así como también se reafirma la hipótesis de que un edificio tiene la capacidad de transformarse, al ser igual de funcional para los diferentes usos que se pueden dar en su interior.

En el aspecto urbano reactivará el sector haciéndolo aún más atractivo y porque genera un movimiento que acarreará más intervenciones en otros tipos de edificaciones pero, seguramente con el mismo enfoque de reutilización y para responder a una necesidad que expresa la sociedad de esta ciudad.

El edificio que por sus características es de vital importancia para la ciudad, amerita con urgencia la necesidad de ser intervenido para que contribuya con su nuevo uso al movimiento que se presenta en la plaza y en los edificios que la circundan. Esta intervención debe hacerse sin perder de vista el valor patrimonial de la edificación y del centro histórico como tal, pero también con una visión contemporánea sobre la interpretación que se da de la historia y del edificio. 


\section{OBJETIVOS}

\section{General}

Reutilizar el edificio del antiguo convento Nuestra Señora del Rosario de Chiquinquirá, dándole un nuevo uso acorde con el contexto actual.

\section{Específicos}

- Integrar el nuevo uso con la vieja estructura, de forma que no se irrespete la obra precedente.

- Restaurar la relación entre el entorno urbano y social, a través de una forma creativa que no destruya el pasado y deje una marca en el tiempo contemporáneo; es decir, permitir que el edificio siga como parte de la memoria colectiva de su sociedad, y del cual se sientan orgullosos tanto por su importancia histórica, como por el resultado que se obtiene del nuevo uso que se genera.

- Diseñar eficazmente espacios que resulten habitables sin dañar la estructura del edificio y que respondan directamente al uso que se le va a dar.

- Resolver el programa arquitectónico en su totalidad, de manera tal que la estructura existente acoja completamente todos los espacios que se necesitan para poder desarrollar de forma apropiada el nuevo uso.

- Establecer una relación directa con su entorno de tipo estético, donde se siga ligado al estilo arquitectónico que pertenece y también de tipo social, que permita la apropiación por parte de la comunidad.

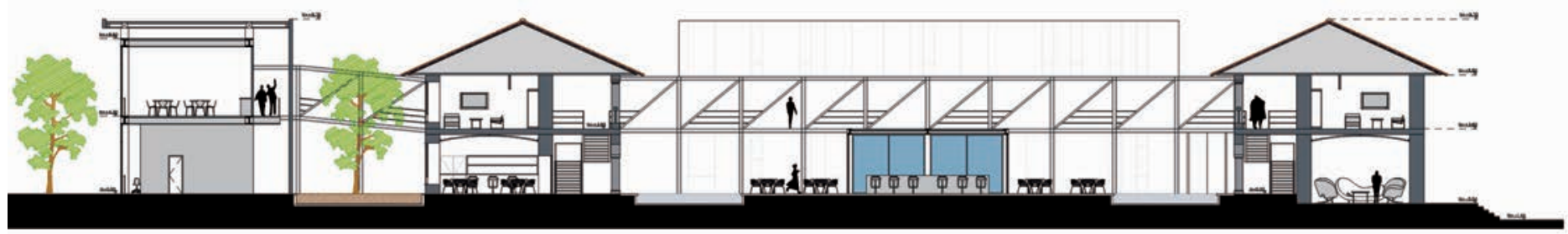

Imagen 3. Corte longitudinal Hotel Convento Nuestra Señora de Chiquinquirá. Fuente: Elaborado por la autora

\section{ESTUDIO DE REFERENTES O TIPOLOGÍAS'}

Los proyectos que fueron analizados se encuentran directamente relacionados con el objetivo del proyecto actual; se trata de intervenciones en edificios que en algún momento de la historia tuvieron un valor para la comunidad donde se encuentran ubicados. El edificio en sí tiene una riqueza espacial y de diseño que permitió que fueran reutilizados y el nuevo uso se integrara perfectamente con la vieja estructura.

En el presente artículo sólo se tuvo en cuenta el estudio de una tipología. 


\section{HOTEL BOUTIQUE LA PURIFICADORA (MÉXICO)}

El proyecto se destaca por la importancia del contexto en el que se encuentra emplazado. Está ubicado en Puebla, México, en el sector que corresponde al centro histórico. La arquitectura que lo rodea se caracteriza por ser en su mayoría de estilo colonial y, por lo tanto, esta determinante lo vuelve privilegiado con respecto a otro edificio en cualquier otro lugar. En sus alrededores se encuentran edificios de gran importancia para la ciudad, como lo son el templo de San Francisco, el museo de San Francisco, un centro comercial y un centro de convenciones, los cuales hacen del sector un espacio atractivo de turismo $y$ un punto de referencia en la ciudad.

En el proyecto se reutilizó un edificio industrial que data de I884, el cual solía ser una fábrica de hielo donde el agua era embotellada y purificada por medio de la producción del hielo, de ahí el nombre del hotel. El edificio de estilo colonial, responde a la arquitectura que lo rodea. La rehabilitación para uso hotelero de la purificadora, forma parte de un proyecto global de renovación del centro histórico de Puebla y sus barrios aledaños.

Por su condición inicial, pues fue proyectado para ser un edificio de carácter industrial, consta originalmente de un gran espacio interior y una envolvente que, a su vez, es la estructura del edificio original, esto permitió, que muchos usos tuvieran cabida, pero finalmente se optó por volverlo un hotel, no tanto por la naturaleza del edificio, sino por el contexto en el cual se encuentra inmerso. De esta forma podemos notar que para tomar la decisión es necesario tener en cuenta tanto la tipología estructural, como el entorno, que en principio es el que condiciona fuertemente cuando proyectamos.

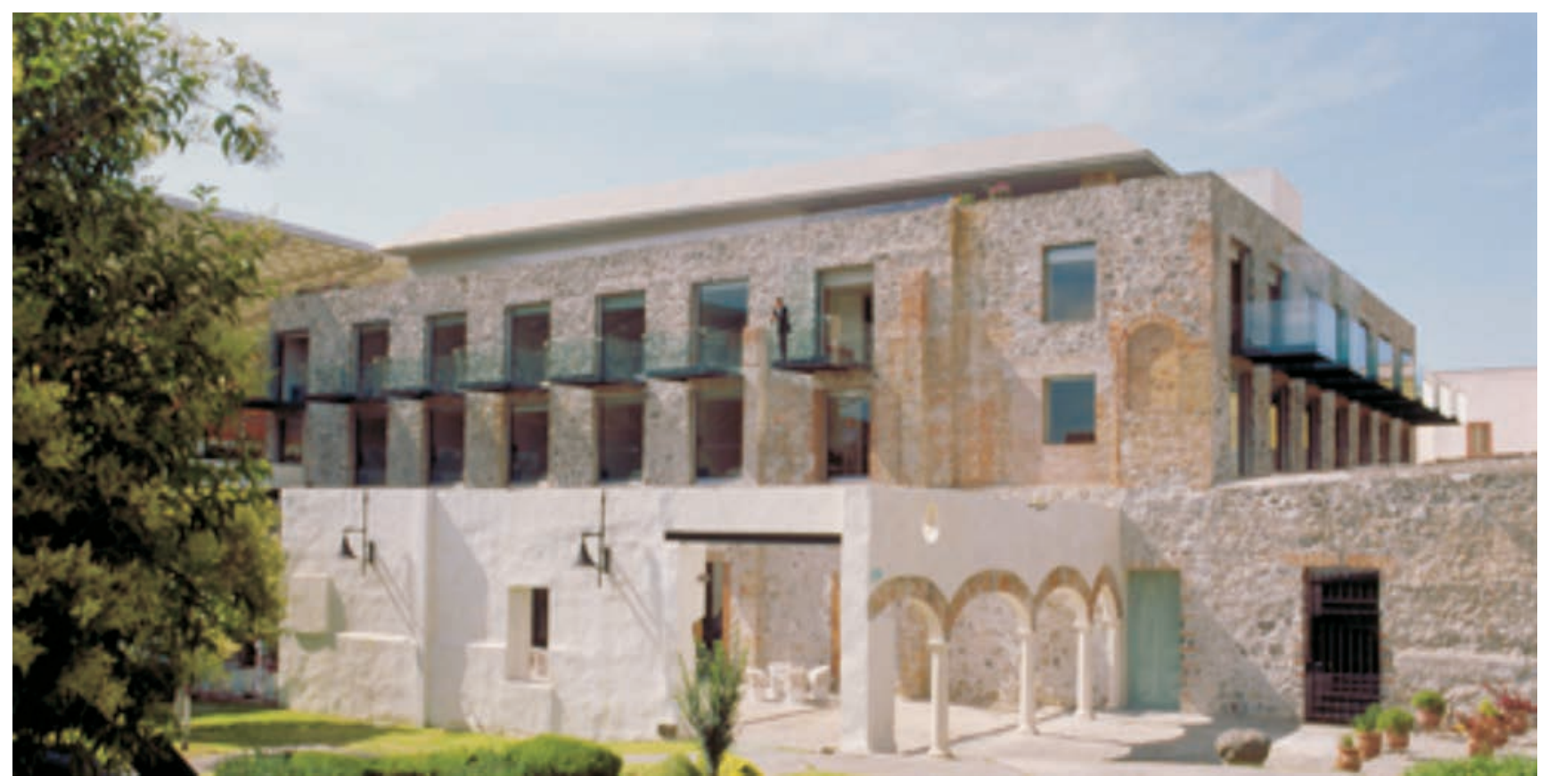




\section{ESTUDIO DE LA LOCALIZACIÓN}

Plano I. Localización del convento. Fuente: Elaborado por la autora.

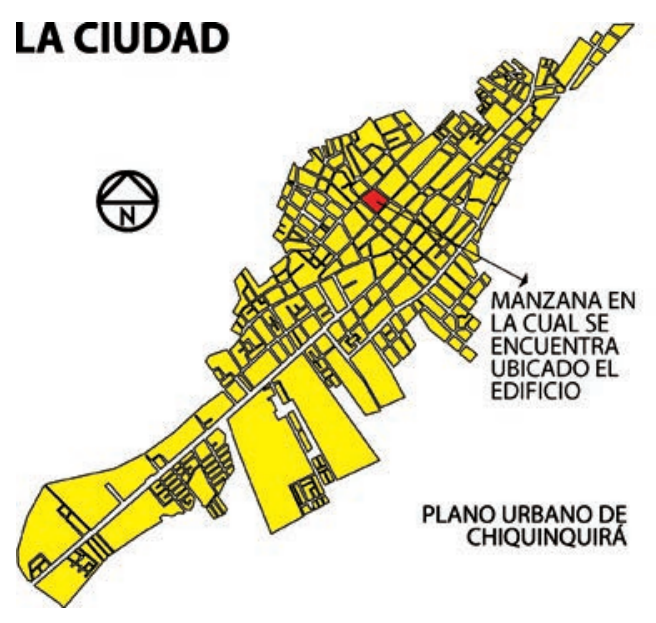

\section{LA MANZANA}

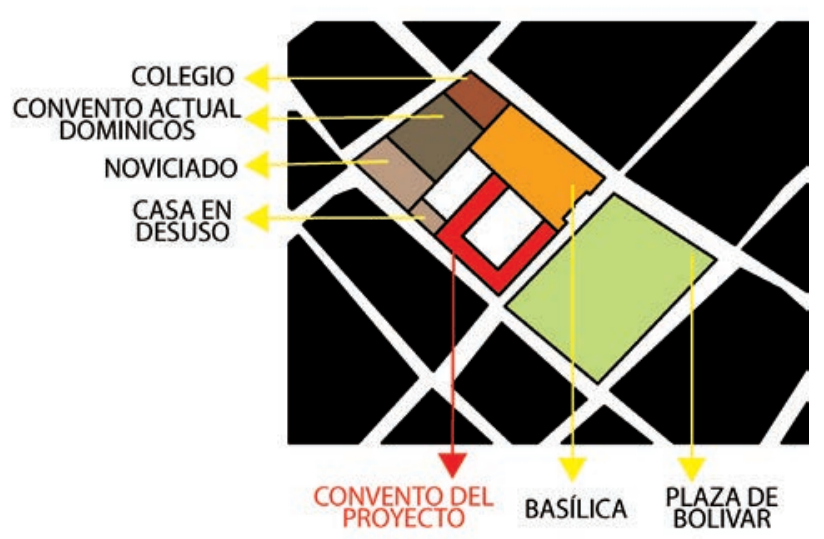

El edificio que se va a reutilizar se encuentra ubicado en Chiquinquirá, municipio al occidente del departamento de Boyacá. La ciudad cuenta con I I5.949 habitantes y se caracteriza por ser un importante nodo comercial de la región a la que provee de ganado y productos agrícolas; pero su mayor reconocimiento en el país y fuera de él, tiene que ver con ser la sede del Santuario Mariano de la Reina de Colombia, nuestra Señora del Rosario de Chiquinquirá.

Chiquinquirá se encuentra en el centro de dos importantes ejes viales, el primero, que conecta a Santafé de Bogotá con Bucaramanga, de importancia nacional; y, el segundo, que conecta al occidente del departamento con el centro y a través de él con todas las demás zonas de importancia regional. Lo anterior, sumado a que gran parte de su desarrollo está relacionado con su condición de ciudad de paso. Las vías Santafé de Bogotá - Bucaramanga y Tunja - Occidente de Boyacá, son al mismo tiempo los principales ejes de conexión urbano - rural del Municipio. 


\section{IMPORTANCIA HISTÓRICA}

El 26 de diciembre de I586, se produjo el milagro de la renovación de la imagen y a partir de la pequeña capilla construida por los indios cocas, creció el pueblo en la medida que llegaron comerciantes en su mayoría europeos o hijos de ellos. En 1640 los padres dominicos iniciaron la construcción del primer convento, en terrenos del hoy parque Julio Flórez. Desde I8I7 este edificio fue la sede del colegio Jesús María y José fundado en I8I3.

Hasta I 794 sólo hubo una plaza, la plaza de la Iglesia de la Virgen; años después se convirtió en la Plaza de la Constitución o parque Julio Flórez. Chiquinquirá fue erigida primero como parroquia en I.588, después como municipio en I.636 y finalmente como ciudad en I.65I. Es conocida como la "Ciudad Mariana de Colombia", allí se venera a la Reina de Colombia cuya imagen se encuentra en la Basílica Mayor ubicada en la Plaza de La Libertad bajo las más estrictas medidas de seguridad por parte de los Frailes Dominicos
Imagen 5. Corte longitudinal 2 Hotel Convento Nuestra Señora de Chiquinquirá. Fuente: Elaborado por la autora

\section{ANÁLISIS DEL SECTOR}

El convento, contiguo a la Basílica de Nuestra Señora de Chiquinquirá, pertenece a una manzana donde también están ubicados el nuevo convento de los Frailes Dominicos y un edificio perteneciente a una comunidad de religiosas. Esta manzana resulta un punto de referencia muy grande en el centro de la ciudad, ya que sobre la fachada principal del convento y de la basílica está ubicada la Plaza de La Libertad la cual genera, en su contexto inmediato, un movimiento importante de tipo religioso, comercial y dotacional. El paisaje urbano predominante en el centro histórico de Chiquinquirá es de tipo comercial pues, poco a poco, este uso se ha ido apropiando de las construcciones que se encuentran allí emplazadas. Se trata en su mayoría de casas de 2 pisos, de diferentes dimensiones en planta, las cuales responden a una distribución característica de la arquitectura colonial.

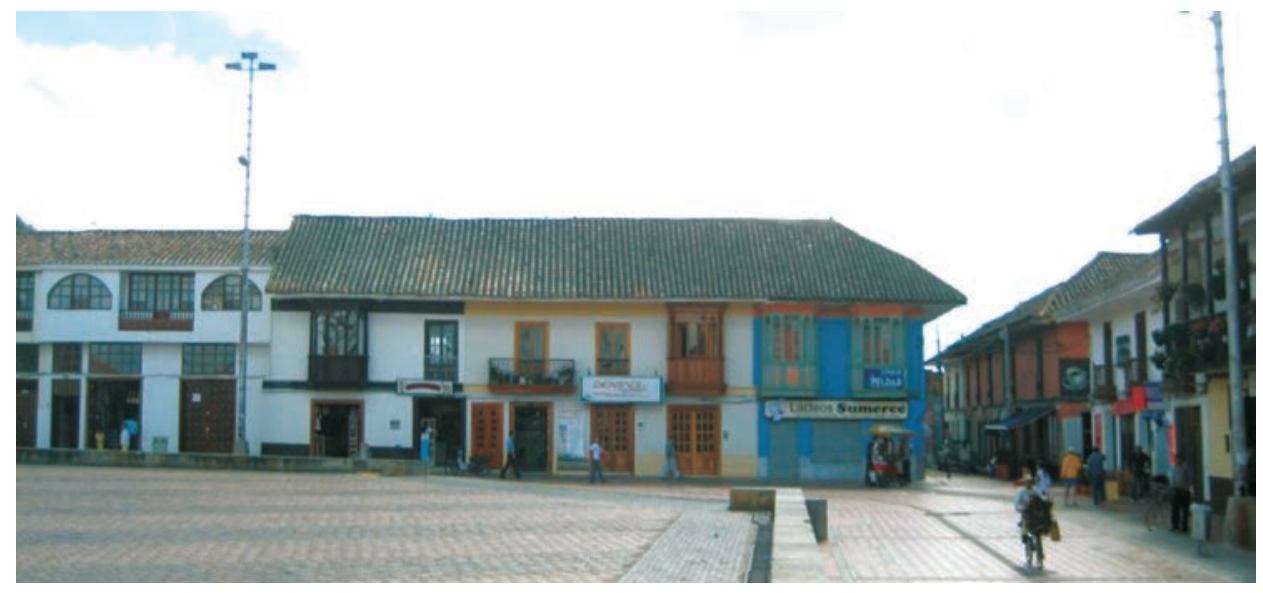

Imagen 6. Alrededores del convento de Chiquinquirá. Fuente: Fotografía de la autora 


\section{ANÁLISIS DE USOS}

De acuerdo al Plan Básico de Ordenamiento Territorial (PBOT) de Chiquinquirá, los usos se ordenan de forma paralela a la vía nacional que une a Bogotá con Bucaramanga, razón por la cual la ciudad ha tenido un desarrollo longitudinal con respecto a esta determinante, habida cuenta que surgió desde un punto céntrico, que ahora se podría identificar como el centro histórico.

Allí el uso residencial envuelve los otros usos, donde los edificios institucionales se ven inmersos sin ningún planeamiento aparente. En los límites de las vías primarias de la ciudad se presenta un cambio de uso, creando mixturas de vivienda y comercio. El pequeño sector industrial se encuentra asentado al sur- oeste y comprimido en un par de cuadras, donde también se combina con vivienda. Por último se puede ver cómo la mancha de comercio se apropió los espacios que antes eran residenciales y los expulsa a la periferia de la ciudad. (Ver plano 2)

Plano 2. Usos del suelo. Fuente: Secretaria de Planeación del municipio

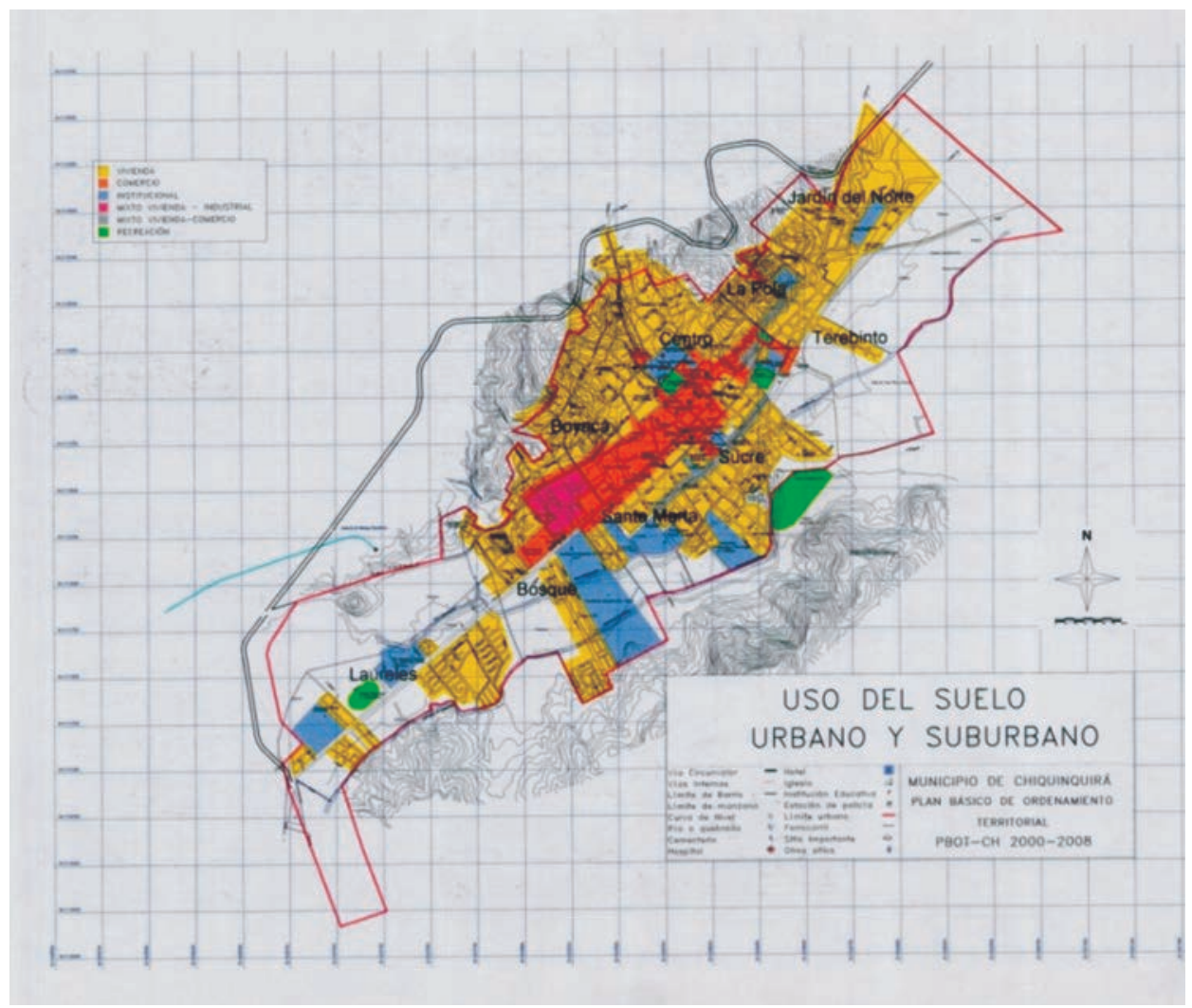

\section{REGLAMENTACIÓN HOTELERA}

La Reglamentación Hotelera dicta las normas que regulan la actividad hotelera colombiana y es, con base en tal documento, que se procede a la clasificación de los hoteles. Cabe resaltar que esta clasificación responde a las características físicas, funcionales, de explotación y de servicios, al igual que a la calidad de la construcción, instalaciones, dotación, equipos, capacitación, presentación de personal y atención que se preste al huésped, condicionantes que hacen que estos establecimientos se dividan en categorías. 


\section{Hoteles}

Se clasifican como hoteles los establecimientos hoteleros o de hospedaje que prestan el alojamiento en unidades habitacionales constituidas por habitaciones y áreas de servicio común y que día a día ofrecen alimentación, servicios básicos y complementarios o accesorios según su modalidad y categoría. Ésta última supone la previa o simultánea clasificación del establecimiento. En el proyecto según la reglamentación hotelera el hotel se presenta como de 3 estrellas.

\section{Programa Tipo}

La reglamentación también expone todos los espacios con los que debe contar el hotel, allí se expresa que el tamaño depende de la cantidad de habitaciones con las que el proyecto cuente.

Después de haber analizado las posibilidades que tiene el convento tanto morfológicas como en su contexto, se decide que la vocación del proyecto será la de un hotel contemporáneo orientado a satisfacer un turismo de tipo religioso, debido a que Chiquinquirá es el Centro Mariano de Colombia, pero también un escenario que presta todos los servicios a los potenciales usuarios (peregrinos y devotos visitantes, amén de turistas) pues su ubicación privilegiada favorecería cualquier tipo de turismo o motivo de viaje.

Para responder a las características del convento, respecto a los tipos de espacios que tiene y sus dimensiones, el proyecto encaja dentro del prototipo de hotel de 3 estrellas. EI reglamento nos presenta un cuadro, en el que se dividen los espacios en 7 grandes grupos, al mostrar cuáles son los espacios obligatorios según las estrellas del hotel, al igual que el área que cada espacio debe tener según el número de habitaciones.

Las áreas presentadas a continuación (Ver Tabla I) se presentan como dimensiones tipo y, por lo tanto, no tienen que ser exactamente las que se apliquen, pero sí marcan una pauta en tamaño para los diferentes espacios. Sin embargo, los espacios reales tienen que estar dentro de los rangos presentados, con posibilidades de variación en porcentajes mínimos.
Imagen 7. Corte transversal Hotel Convento Nuestra Señora de Chiquinquirá.

Imagen 8. Fachada lateral Hotel Convento Nuestra Señora de Chiquinquirá. Fuente: Elaborado por la autora
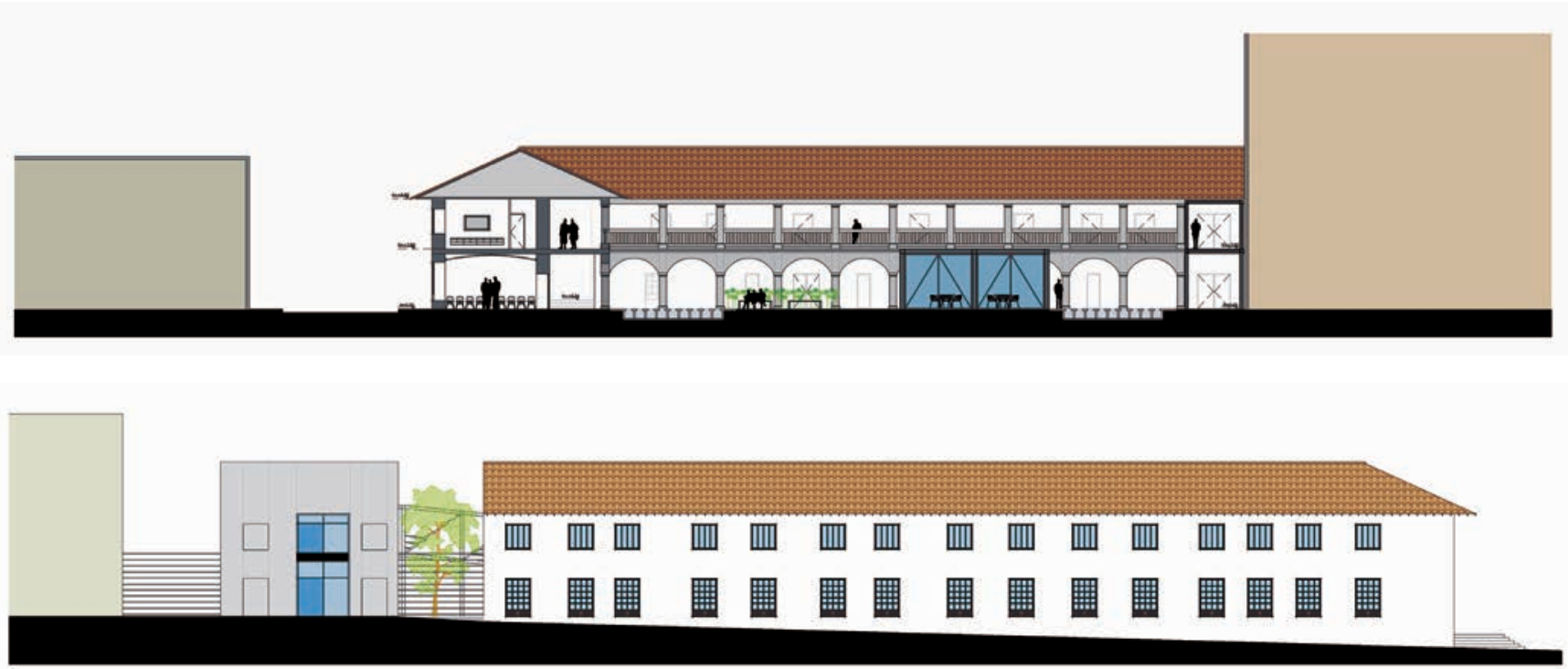
Tabla I. Espacios y áreas del hotel según el reglamento hotelero

\begin{tabular}{|c|c|c|c|}
\hline GRUPOS & ESPACIOS & ÁREA / HAB & 26 HAB \\
\hline \multirow{3}{*}{ I. De los accesos } & I.I Aparcaderos y Garajes & 10 un $(1)$ & \\
\hline & I.2 Entrada de los huéspedes & & \\
\hline & I.3 Entrada de servicio & & \\
\hline \multirow{3}{*}{$\begin{array}{l}\text { 2. De los servicios } \\
\text { de recepción }\end{array}$} & 2.1 Recepción & $1,17 m^{2}$ & $30,42 \mathrm{~m}^{2}$ \\
\hline & 2.2 Vestíbulo & $1,94 m^{2}$ & $50,44 \mathrm{~m}^{2}$ \\
\hline & & & $80,86 m^{2}$ \\
\hline \multirow{2}{*}{$\begin{array}{l}\text { 3. De los servicios } \\
\text { de administración }\end{array}$} & 3.1 Administración & $2,9 m^{2}$ & $75,4 m^{2}$ \\
\hline & & & $75,4 m^{2}$ \\
\hline \multirow{7}{*}{$\begin{array}{l}\text { 4. De los servicios } \\
\text { sociales }\end{array}$} & 4.I Restaurante & $1,8 m^{2}$ & $46,8 m^{2}$ \\
\hline & 4.2 Cafetería & $1,3 m^{2}$ & $33,8 m^{2}$ \\
\hline & 4.3 Bar & $0,9 m^{2}$ & $23,4 m^{2}$ \\
\hline & 4.4 Salón estar & $0,6 m^{2}$ & $15,6 \mathrm{~m}^{2}$ \\
\hline & 4.5 Baños hombres & $0,25 \mathrm{~m}^{2}$ & $6,5 \mathrm{~m}^{2}$ \\
\hline & 4.6 Baños mujeres & $025 m^{2}$ & $6,5 \mathrm{~m}^{2}$ \\
\hline & & & $132,6 \mathrm{~m}^{2}$ \\
\hline \multirow{9}{*}{$\begin{array}{l}\text { 5. De los servicios } \\
\text { generales }\end{array}$} & 5.1 Zona de descargue & $1,2 m^{2}$ & $31,2 m^{2}$ \\
\hline & 5.2 Zona personal & $0,66 \mathrm{~m}^{2}$ & $17,16 \mathrm{~m}^{2}$ \\
\hline & 5.3 Zona de depósitos & $1,7 m^{2}$ & $44,2 m^{2}$ \\
\hline & 5.4 Zona de cocina & $1,8 m^{2}$ & $46,8 m^{2}$ \\
\hline & 5.5 Zona de lavandería & $2,6 m^{2}$ & $67,6 \mathrm{~m}^{2}$ \\
\hline & 5.6 Zona de máquinas & $*(2)$ & \\
\hline & 5.7 Zona de mantenimiento & $1,3 m^{2}$ & $33,8 \mathrm{~m}^{2}$ \\
\hline & 5.8 Otros Servicios & $0,8 m^{2}$ & $20,8 m^{2}$ \\
\hline & & & 261,56 \\
\hline \multirow{3}{*}{$\begin{array}{l}\text { 6. Servicios de } \\
\text { alojamiento }\end{array}$} & 6.I Zonas de alojamiento & $22,45 \mathrm{~m}^{2}$ & $583,7 \mathrm{~m}^{2}$ \\
\hline & 6.2 Zonas de servicio de piso & $1,55 \mathrm{~m}^{2}$ & $40,3 \mathrm{~m} 2$ \\
\hline & & & $624 m^{2}$ \\
\hline
\end{tabular}

(I) Para los parqueaderos son 10 unidades, para un hotel 3 estrellas que tiene entre 10 y 75 habitaciones. (2) El área de la zona de máquinas, estará dada por la cantidad y dimensión de los equipos a instalar.

\section{PROGRAMA ARQUITECTÓNICO}

En respuesta a las condicionantes en que el hotel viene a ocupar espacios ya existentes, el programa no resulta exactamente igual en áreas a lo planteado en la reglamentación, pero respeta todas las dimensiones y cumple con todos los espacios que son obligatorios. 
Tabla 2. Áreas reales del hotel

\begin{tabular}{|c|c|}
\hline \multicolumn{2}{|c|}{ PISO I } \\
\hline \multicolumn{2}{|c|}{ Edificio del convento } \\
\hline I. Administración & $108,91 \mathrm{~m}^{2}$ \\
\hline 2. Recepción y vestíbulo & $101,53 \mathrm{~m}^{2}$ \\
\hline 3. Local I & $37,14 \mathrm{~m}^{2}$ \\
\hline 4. Local 2 & $36,61 \mathrm{~m}^{2}$ \\
\hline 5. Local 3 & $36,61 \mathrm{~m}^{2}$ \\
\hline 6. Local 4 & $36,74 m^{2}$ \\
\hline 7. Local 5 & $42,14 m^{2}$ \\
\hline 8. Baños Hombres I & $24,50 \mathrm{~m}^{2}$ \\
\hline 9. Baños Mujeres I & $16,20 \mathrm{~m}^{2}$ \\
\hline 10. Auditorio I & $154,02 \mathrm{~m}^{2}$ \\
\hline 1I. Auditorio 2 & $136,26 \mathrm{~m}^{2}$ \\
\hline 12. Baño Hombres 2 & $16,70 \mathrm{~m}^{2}$ \\
\hline 13. Baño Mujeres 2 & $18,50 \mathrm{~m}^{2}$ \\
\hline 14. Restaurante-Bar & $167,72 \mathrm{~m}^{2}$ \\
\hline 15. Estar T.V. & $39.00 \mathrm{~m}^{2}$ \\
\hline 16. Depósito & $48,45 \mathrm{~m}^{2}$ \\
\hline 17. Circulaciones & $533,46 \mathrm{~m}^{2}$ \\
\hline \multicolumn{2}{|c|}{ Edificio nuevo } \\
\hline 19. Recepción y vestíbulo & $43,47 m^{2}$ \\
\hline 20. Parqueos & $256,8 m^{2}(1)$ \\
\hline 21. Cocina & $66,14 \mathrm{~m}^{2}$ \\
\hline 22. Vestidores & $17,08 \mathrm{~m}^{2}$ \\
\hline 23. Cuarto Máquinas & $25,55 \mathrm{~m}^{2}$ \\
\hline 24. Circulaciones & $135,8 \mathrm{~m}^{2}$ \\
\hline Total área construida & $2099,33 \mathrm{~m}^{2}$ \\
\hline
\end{tabular}

\begin{tabular}{|c|c|}
\hline \multicolumn{2}{|c|}{ PISO 2} \\
\hline \multicolumn{2}{|c|}{ Edificio del convento } \\
\hline I. Habitaciones & $961,03 \mathrm{~m}^{2}$ \\
\hline 13 hab de $41,17 \mathrm{~m}^{2}$ & \\
\hline 13 hab de $29,11 \mathrm{~m}^{2}$ & \\
\hline 2. Servicios Piso & $30,73 \mathrm{~m}^{2}$ \\
\hline 3. Deposito & $48,45 \mathrm{~m}^{2}$ \\
\hline 4. Circulaciones & $533,46 \mathrm{~m}^{2}$ \\
\hline Edificio nuevo & \\
\hline 5. Lavandería & $63,10 \mathrm{~m}^{2}$ \\
\hline 6. Personal & $61,67 \mathrm{~m}^{2}$ \\
\hline 7. Mantenimiento & $25,55 \mathrm{~m}^{2}$ \\
\hline 8. Circulaciones & $85,62 \mathrm{~m}^{2}$ \\
\hline Total área construida & $1809,61 \mathrm{~m}^{2}$ \\
\hline
\end{tabular}

(I) En total son 8 parqueos y ocupan $256,8 m^{2}$ en área

(2) El patio - claustro tiene un área de $1306,57 \mathrm{~m}^{2}$

Fuente: Elaborado por la autora

PLANTA ALTA

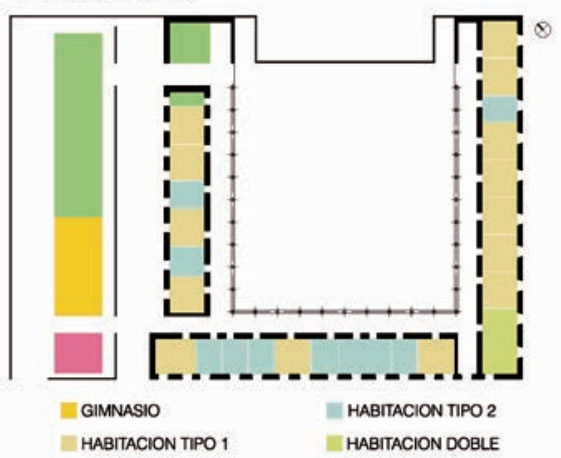

\section{PLANTA BAJA}

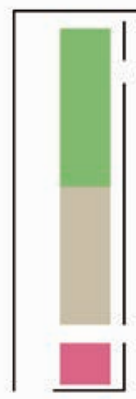

In SERVICIOS IRECEPCION

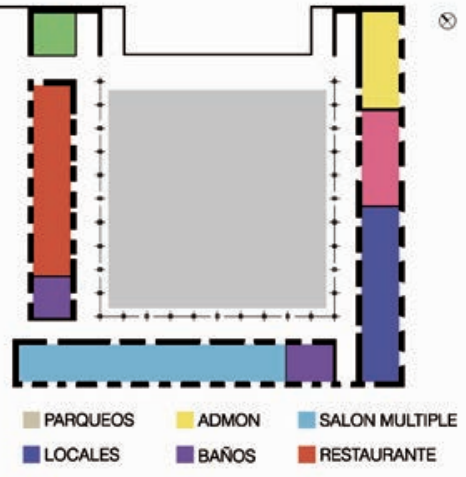

Imagen 9. Zonificaciones. Fuente: Elaborado por la autora 


\section{CONCLUSIONES}

Desde el principio de la investigación se resaltó la importancia de tener una mirada realista de la situación actual del mundo, situación que impulsó el deseo de poder desarrollar un proyecto que fuera consiente y trabajara en paralelo con la posibilidad de contribuir de alguna forma a evitar el desgaste energético y la decadencia de los centros en las ciudades. Este tipo de proyectos aunque poco comunes, son posibles si en el plan para su desarrollo se contemplan aspectos económicos, físicos y sociales.

Cuando se buscó información referente al tema, se hallaron algunos ejemplos a través de los cuales se pudo establecer que estas intervenciones permitieron el rejuvenecimiento de sectores que se pensaba no tenían posibilidad de mejorar. Esta mirada es importante ya que no se puede ver el proyecto sólo como el edificio para reutilizar sino sus alrededores, es decir, el contexto, el sector más inmediato del edificio, que se va a ver beneficiado tanto estética como económicamente, una vez se logren los desarrollos.

De vital importancia resulta en este tipo de proyectos el estudio de su entorno, pues éste arroja información valiosa respecto al uso más adecuado para la edificación, al igual que el tipo de actuaciones que se pueden dar sobre el edificio, tanto interiores como exteriores. Se trata de lograr que el edificio con nuevo uso, se integre de muy buena forma con lo que lo rodea, casi como si este hubiera sido proyectado desde el principio con esta intensión, allí radica el éxito del proyecto, pues permite que sea una inversión rentable y que la comunidad lo acoja como propio.

\section{BIBLIOGRAFÍA}

ALGORRI, Eloy. Un extraño país: el difícil diálogo de la modernidad con el pasado. En: Revista Arquitectura viva, No. I I0, Universidad del Valle, 2006. pp. 26-3I.

GRÛNTUCH, Ernst. Convertible City. Modes of densification and dissolving boundaries.

MONEO, Rafael. Construir lo construido. En: Revista Arquitectura viva, No. I l 0, Universidad del Valle, 2006.

PARICIO, Ignacio. No lo conocerás bastante. En: Revista Arquitectura viva, No. II0,

Imagen 10. Fachada interior Hote Convento Nuestra Señora de Chiquinquirá. Fuente: Elaborado por la autora
Universidad del Valle, 2006.

REVISTA ESCALA No. 7I. Arquitectura para el ocio.

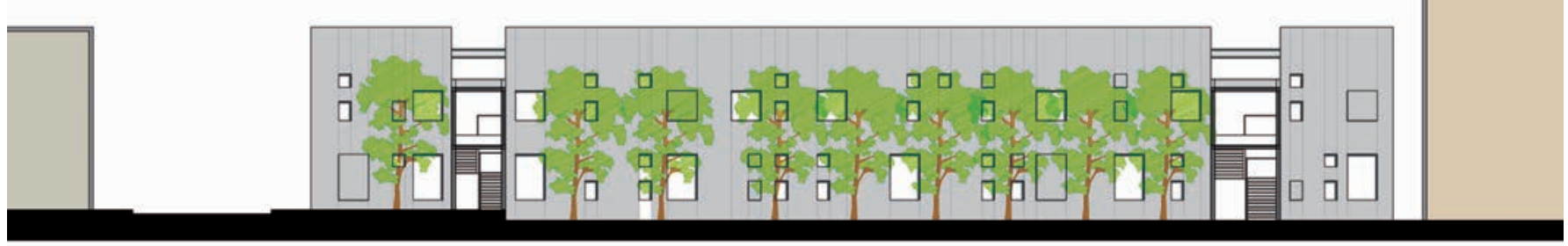




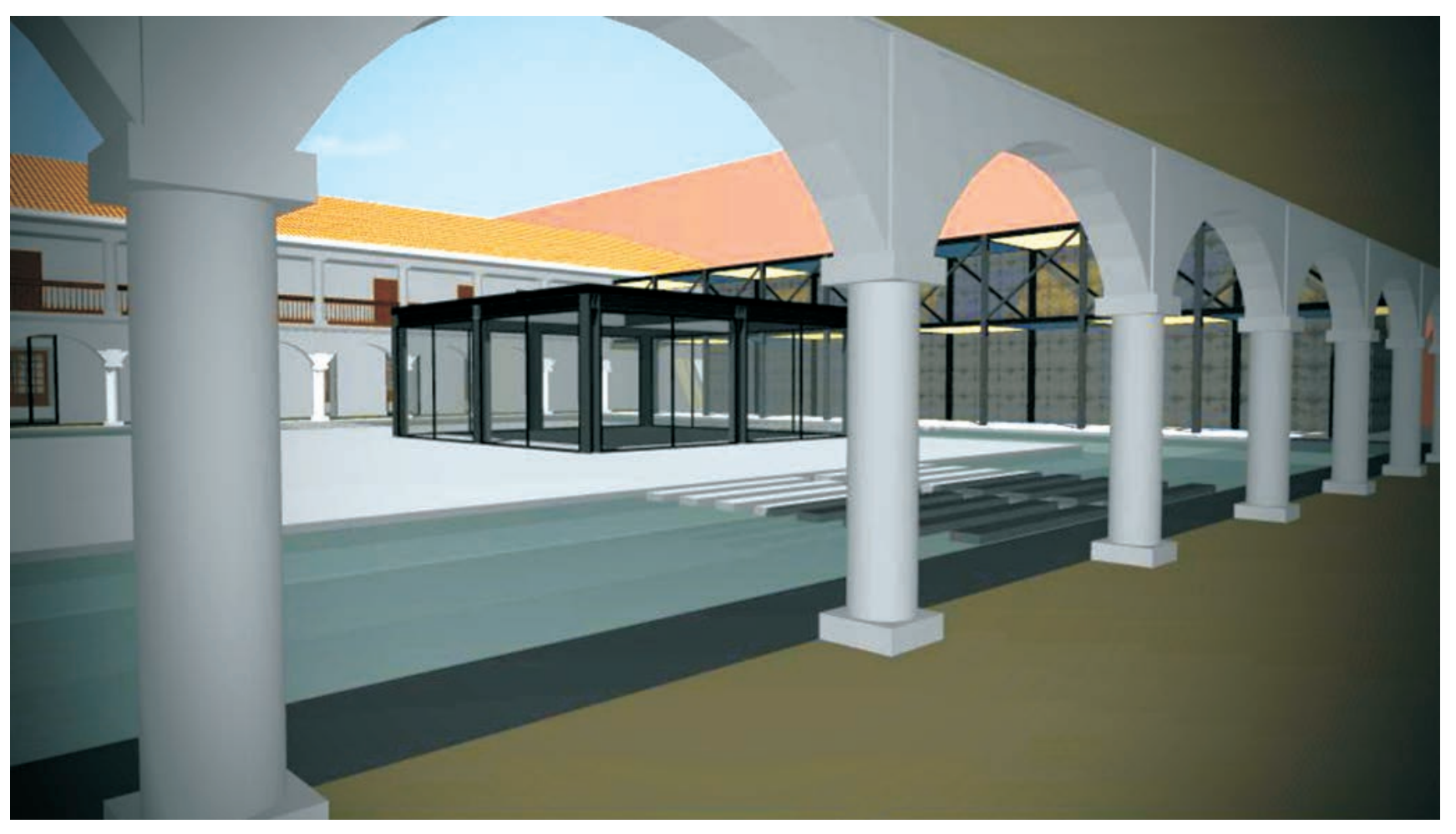

Imagen II. Render vista interior Hotel Convento Nuestra Señora de Chiquinquirá. Fuente: Elaborado por la autora 